\begin{tabular}{|c|c|c|c|c|c|}
\hline \multirow{2}{*}{ Construct } & \multicolumn{5}{|c|}{ Dose-Response Curve Properties } \\
\hline & Leakiness & Fold Change & EC50 & Hill Slope & Area \\
\hline LLL & $3.2 \pm 0.01$ & $31.5 \pm 0.36$ & $4.54 \mathrm{E}-09$ & 1.15 & 150800 \\
\hline LLT & & $32.5 \pm 0.12$ & $1.1 \mathrm{E}-8$ & 0.78 & 144340 \\
\hline LLA & & $29.3 \pm 0.03$ & 4.75E-7 & 0.62 & 81113 \\
\hline LLR & & $1.3 \pm 0.01$ & - & - & 180 \\
\hline LAL & $2.08 \pm 0.02$ & $3.6 \pm 0.45$ & $\geq 5 \mathrm{E}-5$ & - & 644 \\
\hline LAT & & $26.9 \pm 0.26$ & $2.08 \mathrm{E}-06$ & 1.13 & 35200 \\
\hline LAA & & $28.6 \pm 0.42$ & $1.34 \mathrm{E}-08$ & 0.69 & 83782 \\
\hline LAR & & $2.5 \pm 0.28$ & $\geq 5 \mathrm{E}-5$ & - & 425 \\
\hline LRL & $2.52 \pm 0.01$ & $3.1 \pm 0.40$ & $\geq 5 \mathrm{E}-5$ & - & 402 \\
\hline LRT & & $2.7 \pm 0.07$ & $\geq 5 \mathrm{E}-5$ & - & 546 \\
\hline LRA & & $1.1 \pm 0.01$ & - & - & 18 \\
\hline LRR & & $29.9 \pm 0.22$ & 1.37E-09 & 1.14 & 131890 \\
\hline TTL & $1.74 \pm 0.01$ & $5.1 \pm 0.06$ & $5.89 \mathrm{E}-08$ & 1.01 & 8235 \\
\hline TTT & & $5.4 \pm 0.06$ & $3.16 \mathrm{E}-08$ & 1.19 & 9390 \\
\hline TTA & & $7.1 \pm 0.05$ & $9.65 \mathrm{E}-7$ & 0.86 & 8067 \\
\hline TTR & & $1.1 \pm 0.01$ & - & - & 43 \\
\hline AAL & $3.93 \pm 0.01$ & $15.4 \pm 2.35$ & $\geq 5 \mathrm{E}-5$ & - & 5003 \\
\hline AAT & & $90.3 \pm 0.52$ & $5.95 \mathrm{E}-7$ & 1.29 & 279910 \\
\hline AAA & & $98.7 \pm 1.11$ & $1.18 \mathrm{E}-09$ & 0.94 & 689740 \\
\hline AAR & & $8.1 \pm 1.55$ & $\geq 5 \mathrm{E}-5$ & - & 2639 \\
\hline RLL & $2.40 \pm 0.01$ & $64.5 \pm 0.51$ & $3.15 \mathrm{E}-7$ & 0.45 & 156500 \\
\hline RLT & & $63.0 \pm 0.17$ & 2.57E-06 & 0.41 & 122680 \\
\hline RLA & & $19.6 \pm 0.16$ & $2.00 \mathrm{E}-03$ & 0.41 & 20147 \\
\hline RLR & & $1.0 \pm 0.0$ & - & - & 53 \\
\hline RAL & $2.28 \pm 0.04$ & $6.6 \pm 0.94$ & $\geq 5 \mathrm{E}-5$ & - & 1223 \\
\hline RAT & & $59.8 \pm 0.43$ & $1.03 \mathrm{E}-06$ & 1.12 & 93241 \\
\hline RAA & & $62.6 \pm 1.45$ & 8.39E-10 & 0.58 & 241590 \\
\hline RAR & & $3.7 \pm 0.54$ & $\geq 5 \mathrm{E}-5$ & - & 641 \\
\hline RRL & $5.71 \pm 0.07$ & $7.0 \pm 0.73$ & $\geq 5 \mathrm{E}-5$ & - & 3551 \\
\hline RRT & & $6.7 \pm 0.11$ & $\geq 5 \mathrm{E}-5$ & - & 3839 \\
\hline RRA & & $1.9 \pm 0.20$ & $\geq 5 \mathrm{E}-5$ & - & 1036 \\
\hline RRR & & $27.7 \pm 0.12$ & $4.99 \mathrm{E}-10$ & 1.299 & 306560 \\
\hline
\end{tabular}

Table S1 Characteristics of QS circuit dose-responses. Each promoter-R-protein pair has the same leakiness regardless of HSL, so it is only listed once. EC50 was not estimated for constructs with fold changes lower than 2, and any construct that exhibited a greater than 2-fold change only at 100uM was assumed to have an EC50 greater than or equal to $50 \mathrm{uM}$. Hill slopes were only calculated for constructs that showed significant activation before $100 \mathrm{uM}$. 


\begin{tabular}{|c|c|c|c|}
\hline Name & Sequence & Organism & Reference \\
\hline Plux & $\begin{array}{l}\text { ACTATTGTATCGCTGGGAATACAATTACTTAACATAAGCAC } \\
\text { CTGTAGGATCGTACAGGTTTACGCAAGAAAATGGTTTGTT } \\
\text { ATAGTCGAATAT }\end{array}$ & Aliivibrio fischeri & (Hasty Lab) \\
\hline $\begin{array}{l}\text { Ptra } \\
\text { (pCF370) }\end{array}$ & $\begin{array}{l}\text { CTACGTGCAGATCTGCACATAGCCACACCCTGAATGAGATG } \\
\text { TTTTCTCTCCGCTA }\end{array}$ & $\begin{array}{l}\text { Agrobacterium } \\
\text { tumefaciens }\end{array}$ & $\begin{array}{l}\text { (54) White } \\
\text { 2007, (53) } \\
\text { Fuqua 1996 }\end{array}$ \\
\hline Plas & $\begin{array}{l}\text { TTCGAGCCTAGCAAGGGTCCGGGTTCACCGAAATCTATCTC } \\
\text { ATTTGCTAGTTATAAAATTATGAAATTTGCGTAAATTCTT } \\
\text { CA }\end{array}$ & $\begin{array}{l}\text { Pseudomonas } \\
\text { aeruginosa }\end{array}$ & (Hasty Lab) \\
\hline Prpa & $\begin{array}{l}\text { ACCTGTCCGATCGGACAGTAGTTAGGTTCCCGTTCGCACCT } \\
\text { GCACTGTTCCCGCCTGCA }\end{array}$ & $\begin{array}{l}\text { Rhodopseudomonas } \\
\text { palustris }\end{array}$ & $\begin{array}{l}\text { (56) Hirakawa } \\
2011\end{array}$ \\
\hline Pahy & $\begin{array}{l}\text { ACCGAAGTGAAATGTTCGAGGTACTCAAGCAGTTGGTCTTG } \\
\text { TTTCATATGCTAGCCCCCCTGGCCAGGGCCTCGATTATA }\end{array}$ & $\begin{array}{l}\text { Aeromonas } \\
\text { hydrophila }\end{array}$ & $\begin{array}{l}\text { (61) Garde } \\
2010\end{array}$ \\
\hline Psma & $\begin{array}{l}\text { ATAATCTTGTCATGGGTTTTAAATTTACTTGTCACATAGGC } \\
\text { TCTGATACAATTACTCGCCG }\end{array}$ & Serratia marcescens & $\begin{array}{l}\text { (62) Slater } \\
2003\end{array}$ \\
\hline $\begin{array}{l}\text { Prhl } \\
\text { (qscrhlA) }\end{array}$ & $\begin{array}{l}\text { TCCTGTGAAATCTGGCAGTTACCGTTAGCTTTCGAATTGG } \\
\text { CTAAAAAGTGTTC }\end{array}$ & $\begin{array}{l}\text { Pseudomonas } \\
\text { aeruginosa }\end{array}$ & $\begin{array}{l}\text { (63) Karig } \\
2005\end{array}$ \\
\hline $\begin{array}{l}\text { Pcer } \\
\text { (PopgG) }\end{array}$ & $\begin{array}{l}\text { GCTGGACGATGCGAATCTTGGAATTGCGCTCTGCAAGCCAT } \\
\text { TGAAAAACGGACGTCGTCTCTCTGATATGCCCGCTCCTGCCG } \\
\text { CCCCCTCCGCCCGCCTGAACCGGCGCCTGCTGCTCAGCGCGG } \\
\text { CAAGTTCGTCGCTCGCCCTCGCTGCAAGCGGGCTC }\end{array}$ & $\begin{array}{l}\text { Rhodobacter } \\
\text { sphaeroides }\end{array}$ & $\begin{array}{l}\text { (59) Puskas } \\
1997\end{array}$ \\
\hline $\begin{array}{l}\text { Pexp } \\
\text { (PwgeA) }\end{array}$ & $\begin{array}{l}\text { TGCCTGCATTTCCGTCAGAATTACTCCTAAAATTATATTG } \\
\text { TACCAATATTGGCACAGCATGGAGATATGTTTCGGGCACCC } \\
\text { TCTTTCTATCAAAATATCGCCGTTTTATTTTATGCATCTGTG } \\
\text { TTGCGTTCGTAATTATTGCAGTGCACACTCCGGCACG }\end{array}$ & $\begin{array}{l}\text { Sinorhizobium } \\
\text { meliloti }\end{array}$ & $\begin{array}{l}\text { (60) } \\
\text { Charoenpanich } \\
2013\end{array}$ \\
\hline Ptra* & $\begin{array}{l}\text { GCACGTGCAGATCTGCACATTTACGCAAGAAAATGGTTTG } \\
\text { TTATAGTCGAATAT }\end{array}$ & Synthetic & This Study \\
\hline Prpa* & $\begin{array}{l}\text { GCACCTGTCCGATCGGACAGTATTACGCAAGAAAATGGTT } \\
\text { TGTTATAGTCGAATAT }\end{array}$ & Synthetic & This Study \\
\hline
\end{tabular}

Table S2 Promoter sequences used in this study. Palindromic lux-box-like receptor-binding sequences in boldface when known. 


\begin{tabular}{|c|c|c|}
\hline Plasmid & Genotype & Vector \\
\hline Bsrs079-LuxR & R6K; Spec; 5' Insulation Unit; BCD7; LuxR-GFP; 3' Insulation Unit & pBjk2807 \\
\hline Bsrs079-LasR & R6K; Spec; 5' Insulation Unit; BCD7; LasR-GFP; 3' Insulation Unit & pBjk2807 \\
\hline Bsrs079-TraR & R6K; Spec; 5' Insulation Unit; BCD7; TraR-GFP; 3' Insulation Unit & pBjk2807 \\
\hline Bsrs079-RpaR & R6K; Spec; 5' Insulation Unit; BCD7; RpaR-GFP; 3' Insulation Unit & pBjk2807 \\
\hline Bsrs079-RhIR & R6K; Spec; 5' Insulation Unit; BCD7; RhIR-GFP; 3' Insulation Unit & pBjk2807 \\
\hline Bsrs079-SinR & R6K; Spec; 5' Insulation Unit; BCD7; SinR-GFP; 3' Insulation Unit & pBjk2807 \\
\hline Bsrs079-CerR & R6K; Spec; 5' Insulation Unit; BCD7; CerR-GFP; 3' Insulation Unit & pBjk2807 \\
\hline Bsrs079-SmaR & R6K; Spec; 5' Insulation Unit; BCD7; SmaR-GFP; 3' Insulation Unit & pBjk2807 \\
\hline Bsrs079-AhyR & R6K; Spec; 5' Insulation Unit; BCD7; AhyR-GFP; 3' Insulation Unit & pBjk2807 \\
\hline Bsrs078-LuxR & R6K; Spec; 5' Insulation Unit; BCD7; LuxR; 3' Insulation Unit & pBjk2807 \\
\hline Bsrs078-TraR & R6K; Spec; 5' Insulation Unit; BCD7; TraR; 3' Insulation Unit & pBjk2807 \\
\hline Bsrs078-TraR(W) & R6K; Spec; 5' Insulation Unit; BCD7; TraR(W); 3' Insulation Unit & pBjk2807 \\
\hline Bsrs078-LasR & R6K; Spec; 5' Insulation Unit; BCD7; LasR; 3' Insulation Unit & pBjk2807 \\
\hline Bsrs078-RpaR & R6K; Spec; 5' Insulation Unit; BCD7; RpaR; 3' Insulation Unit & pBjk2807 \\
\hline Bsrs074-Plux & ColE2; AmpR; Plux; u6; sfGFP & pBjk2992 \\
\hline Bsrs074-Ptra & ColE2; AmpR; Ptra; u6; sfGFP & pBjk2992 \\
\hline Bsrs074-Ptra* & ColE2; AmpR; Ptra*; u6; sfGFP & pBjk2992 \\
\hline Bsrs074-Plas & ColE2; AmpR; Plas; u6; sfGFP & pBjk2992 \\
\hline Bsrs074-Prpa & ColE2; AmpR; Prpa; u6; sfGFP & pBjk2992 \\
\hline Bsrs074-Prpa* & ColE2; AmpR; Prpa*; u6; sfGFP & pBjk2992 \\
\hline Bsrs074-Pahy & ColE2; AmpR; Pahy; u6; sfGFP & pBjk2992 \\
\hline Bsrs074-Psma & ColE2; AmpR; Psma; u6; sfGFP & pBjk2992 \\
\hline Bsrs074-Prhl & ColE2; AmpR; Prhl; u6; sfGFP & pBjk2992 \\
\hline Bsrs074-Pcer & ColE2; AmpR; Pcer; u6; sfGFP & pBjk2992 \\
\hline Bsrs074-Pexp & ColE2; AmpR; Pexp; u6; sfGFP & pBjk2992 \\
\hline Bsrs074-Plux-mKate2 & ColE2; Cm; Plux; u6; mKate2 & pBjk2992 \\
\hline Bsrs074-Plas-mKate2 & ColE2; Cm; Plux; u6; mKate2 & pBjk2992 \\
\hline pZA35-Bsrs074-Plux-tra & P15A; Cm; Ptra; u6; sfGFP & pZA35 \\
\hline Bsrs112-TraR-RpaR & R6K; Spec; 5' Insulation Unit; BCD7; TraR(W); mevB-rbs; RpaR; 3' Insulation Unit & pBjk2807 \\
\hline Bsrs112-TraR-LasR & R6K; Spec; 5' Insulation Unit; BCD7; TraR(W); mevB-rbs; LasR; 3' Insulation Unit & pBjk2807 \\
\hline Bsrs103-RpaR-Rpal & ColE1; Kan; RpaR; Pluxl; Rpal; T1 & pTD103 \\
\hline Bsrs103-RpaR-Lasl & ColE1; Kan; RpaR; Pluxl; Lasl; T1 & pTD103 \\
\hline
\end{tabular}

Table S3 Plasmids used in this study. All constructs were expressed in E. coli DIAL Strain "EK" cells. 


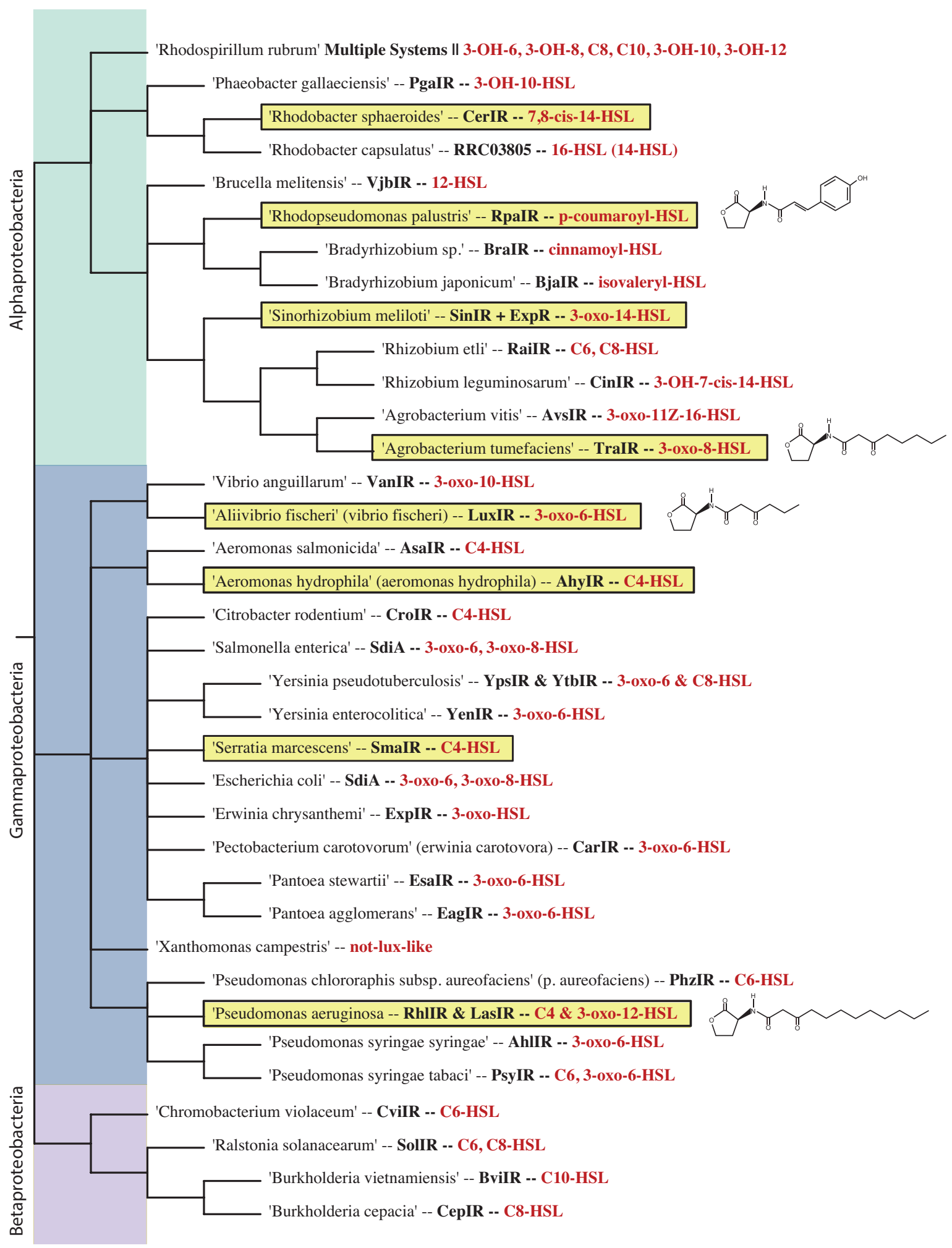

Fig. S1 Evolutionary tree of selected Lux-like QS systems. Systems selected for initial analysis are highlighted in yellow. Each system's HSL is written in red; the chemical structure of the four HSL's particular to the systems characterized in this study are shown on the far right. Potential systems were chosen based on evolutionary distance, ligand uniqueness, and available information in the literature on their potential for recombinant expression 


\section{Promoter-Receptor Pair Leakiness}

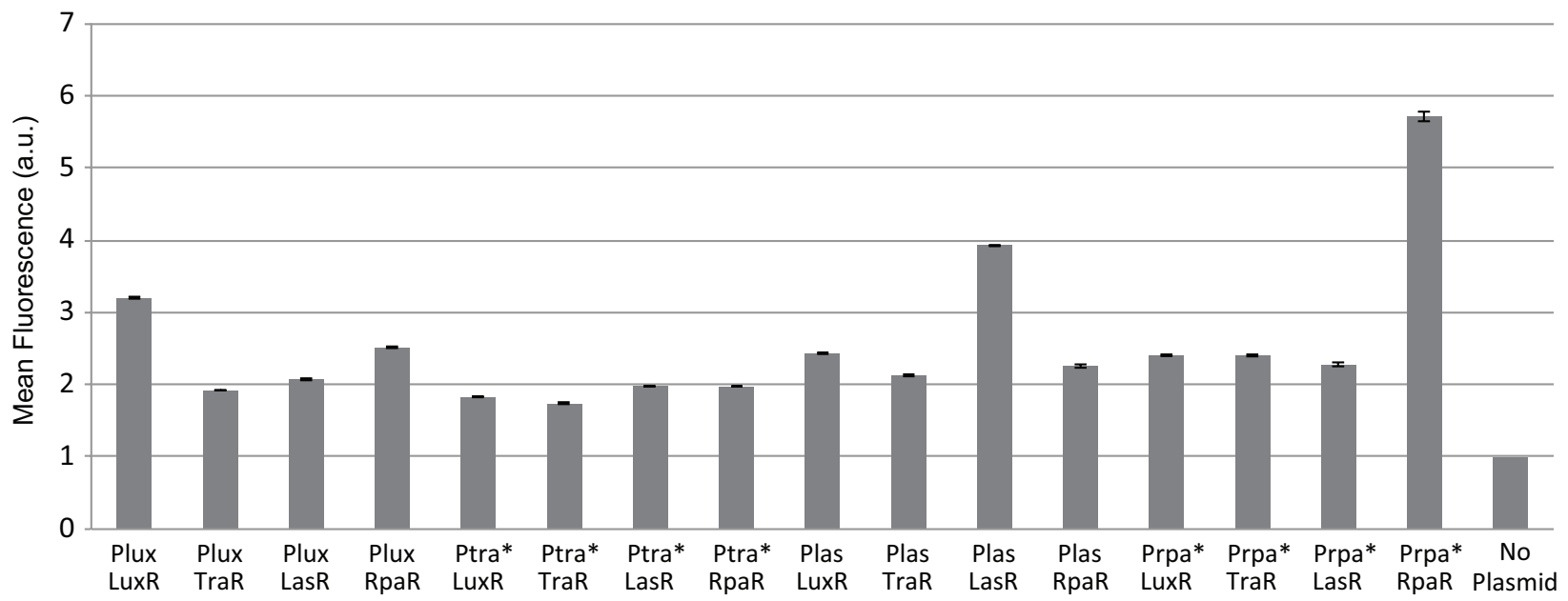

Fig. S2 Promoter/Receptor Pairs Exhibit Differential Basal Expression Levels. Mean fluorescence of Receptor/Reporter twoplasmid systems in the absence of any HSL signal. All values are normalized by the auto-fluorescence of the same E. coli strain with no GFP plasmid. Error bars represent S.E.M (n=3).

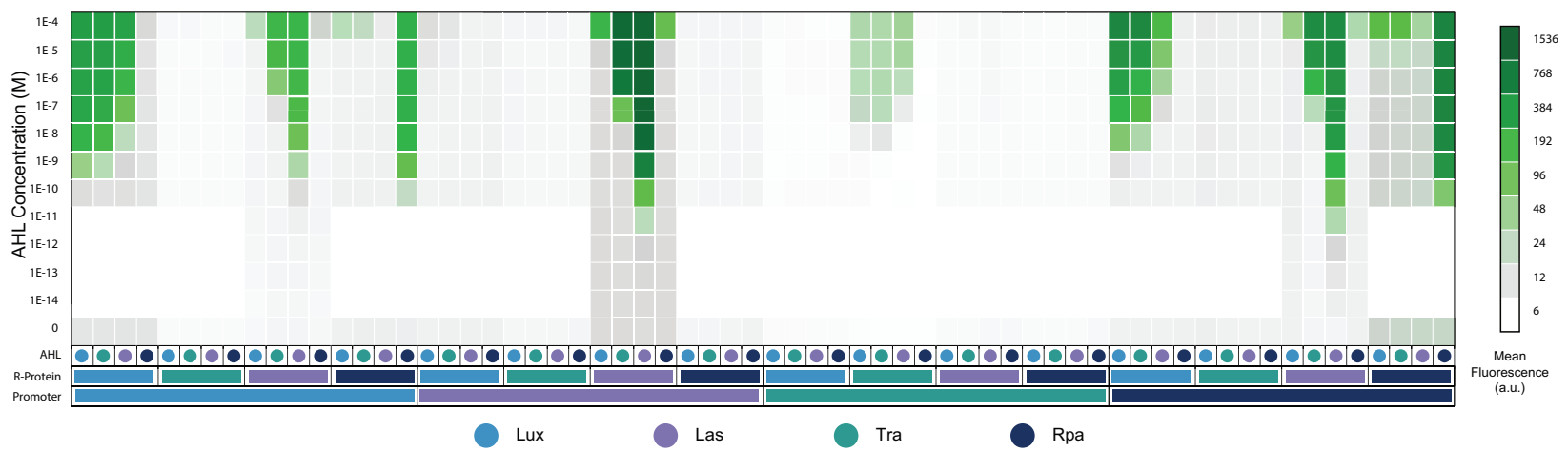

Fig. S3 Heat map showing GFP abundance for all QS combinations and HSL concentrations. Each column denotes a unique combination of signal (HSL), receptor (R- protein), and reporter (QS promoter), with rows denoting the concentration of ligand. Each value corresponds to the mean fluorescence value measured from a cell population normalized by the population's celldensity; all combinations and concentrations were done in triplicate.

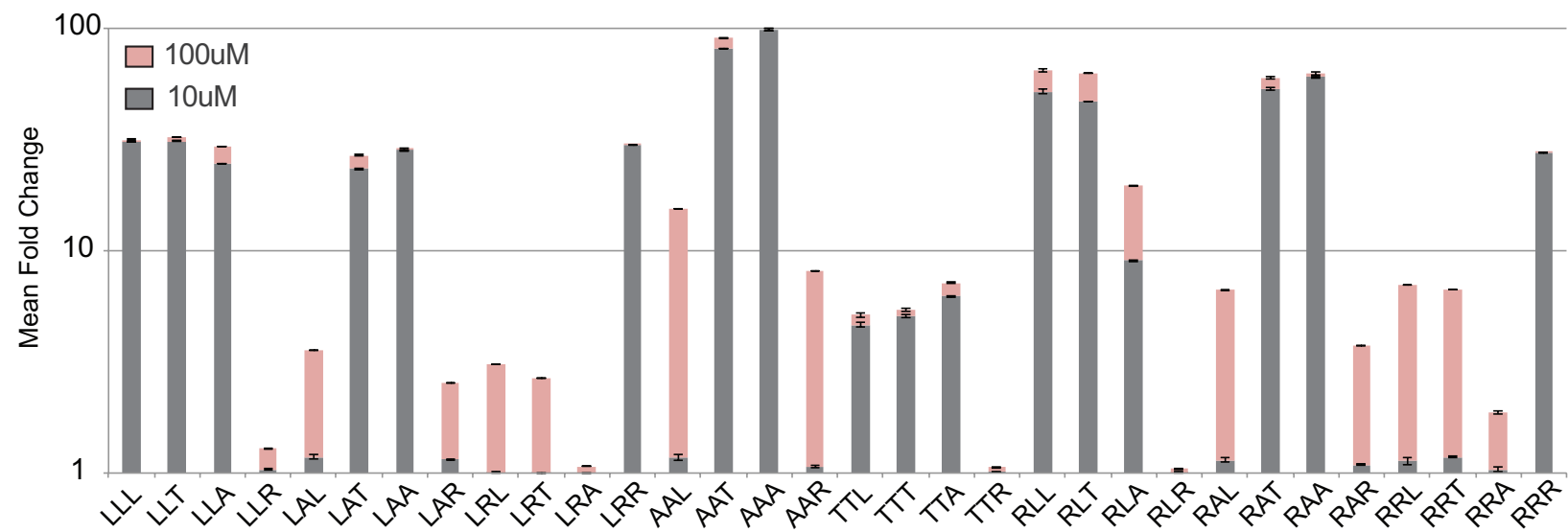

Fig. S4 Large Increase in Expression at 100uM Distorts Relative Performance of Constructs. Fluorescence value at 10uM and 100uM divided by basal expression rate in absence of HSL to give Fold Change, error bars denote SEM ( $n=3$ ). Many systems still show little to no expression at the already very high ligand concentration of $10 \mathrm{uM}$, but exhibit a large jump in expression at $100 \mathrm{uM}$. Since this concentration value is thought to be physiologically irrelevant, fold change based off that concentration can inaccurately describe promoter performance. 

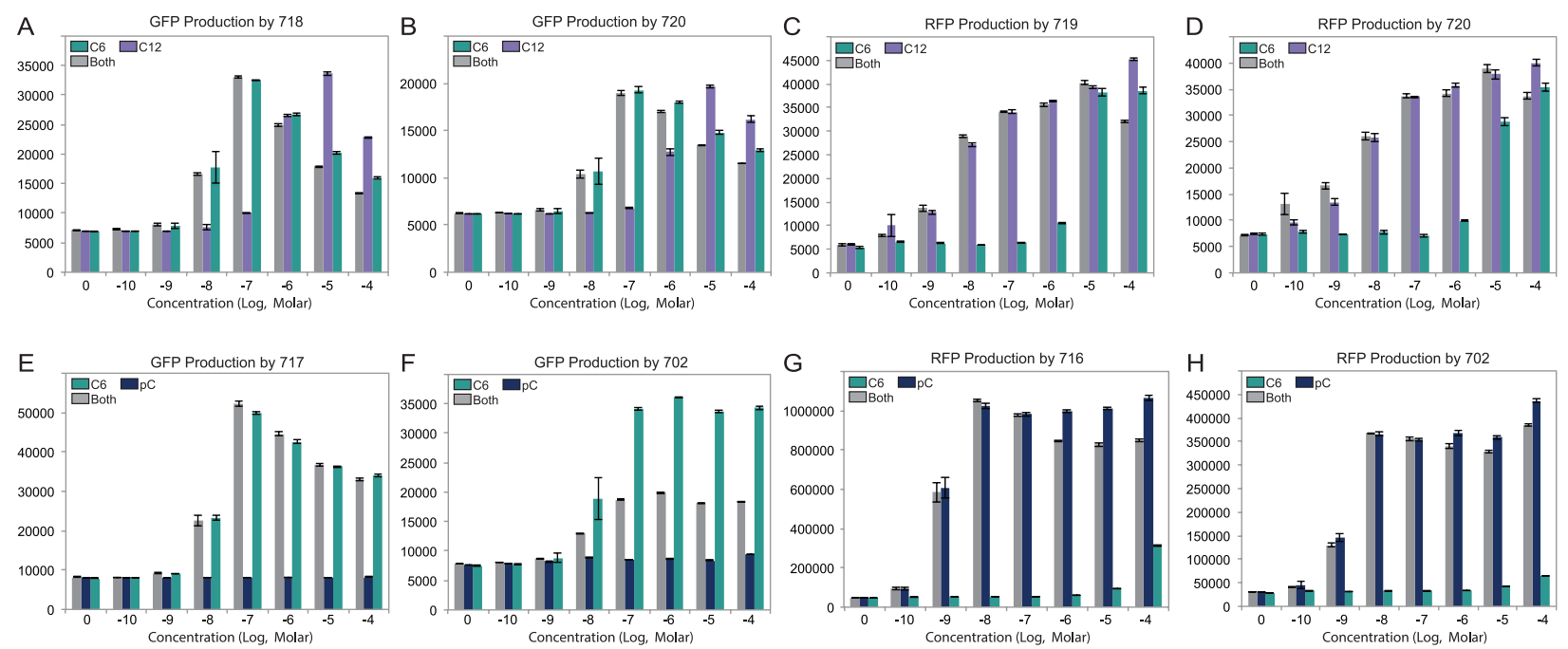

Fig. S5 Side-by-side comparison of raw fluorescence expression between 2-plasmid and 3-plasmid orthogonality strains. (AD) Raw expression from promoter orthogonal strains 718, 717, and 720. (E-H) Raw expression fro complete orthogonal strains 717, 716, and 702. Expression from Strain 717 with C6 and Both is almost identical, however from Strain 702, Both HSLs result in significantly less GFP which is likely a result of growth defects caused by metabolic load of producing RFP at the same time. Error bars represent S.E.M $(n=4)$.
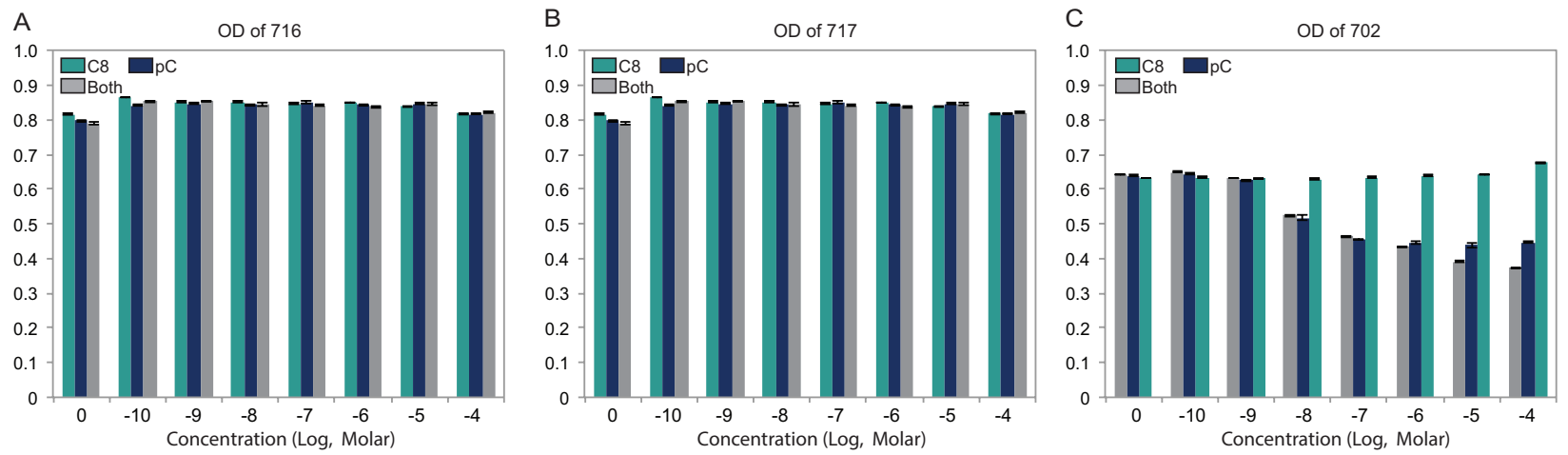

Fig. S6 Growth defects in the 3-plasmid "Complete Orthogonality" strain 702 compared to the 2-plasmid complete orthogonality strains of 716 and 717. (A-B) OD of strain 716 and 7173 hours after induction with HSLs. No apparent change in OD due to HSL concentration. (C) Base level OD of Strain 702 is less, likely due to harboring three relative high-copy plasmids. Furthermore, OD decreases with increased concentrations of pC-HSL, likely due to growth defects caused by the production of RFP. These growth defects can distort the meaning of OD-normalized dose-responses. Error bars represent S.E.M $(n=4)$.

A

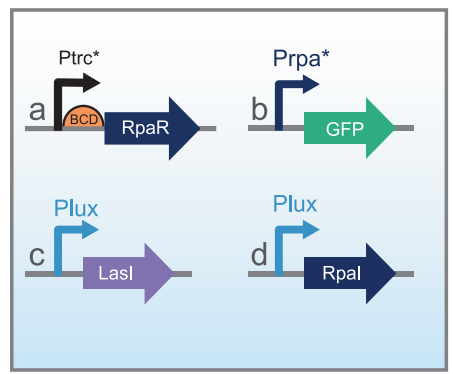

$\mathbf{R}=a+b \quad R R-A i=a+b+c R-R i=a+b+d$
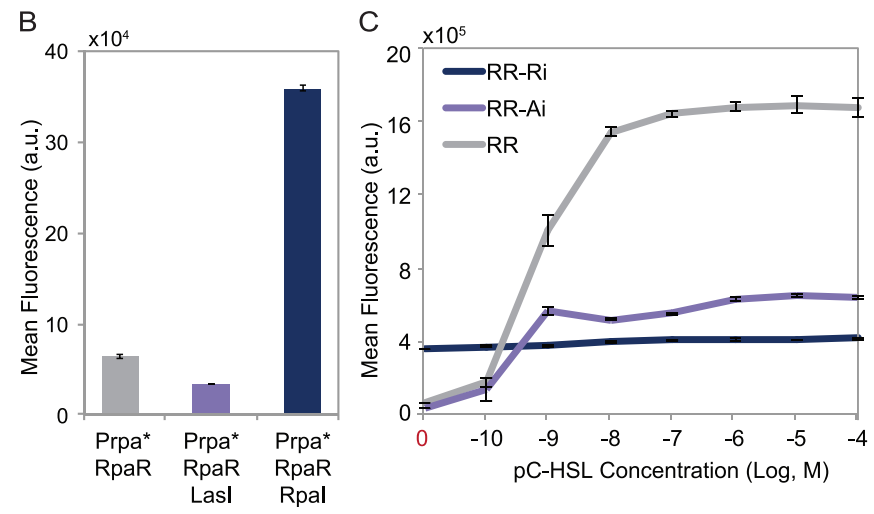
Fig. S7 (B) Inclusion of an RpaI expressing plasmid causes increased GFP expression in an RpaR-expressing strain with GFP driven by the Prpa* promoter. Error bars represent S.D. $(n=4)$. (C) Exogenously providing HSL to the strain with RpaI shows little induction at even the highest concentration of $100 \mathrm{uM}$, hinting that RpaI alone saturates the production capabilities. Raw expression is likely less in the 3-plasmid systems for the same metabolic reasons previously described. Error bars represent S.D. $(n=4)$. 